\title{
Selection of Lodging Resistant Lines in Early Generations Using Linked Molecular Markers and Phenotypic Traits in Rice
}

\author{
M. Girija Rani* and P.V. Satyanarayana
}

Acharya N G Ranaga Agricultural University, Andhra Pradesh Rice Research Institute and Regional Agricultural research Station, Maruteru-534122, West Godavari district, India

*Corresponding author

\section{A B S T R A C T}

\begin{tabular}{|l|}
\hline K e y w o r d s \\
Rice, Lodging \\
resistance, Molecular \\
markers, Selection, \\
Early generations
\end{tabular}

Rice crop in cyclone prone coastal areas is most frequently subjected to lodging at reproductive stage causing severe yield loss. Breeding for non-lodging rice varieties is herculean task as it is controlled by structural properties of the culm and weather parameters. Present study aimed to select lodging resistant lines using phenotypic characters and molecular markers in early generations. Co-segregation banding pattern for lodging resistant families with either homozygous donor allele or heterozygous allele for markers RM 20557, RM 5509 among the $F_{3}$ families of MTU 7029/II 110-9-1-1-1-1 and for markers RM 6933 and RM 216 in case of $F_{3}$ families pertaining to MTU 7029/MTU1121 indicates that these markers can be useful in selection of lodging resistant lines in early generations. Correlation studies revealed that positive correlation of culm diameter with panicle length was observed among the $F_{3}$ families of MTU 7029/II 110-91-1-1-1 and negative correlation of bending strength with number of ear bearing tillers per plant in case of MTU 7029/MTU 1121 indicates specific contribution of alleles for lodging resistance depends on donors used.

\section{Introduction}

Lodging of rice crop is one of the constraints to rice productivity in cyclone prone coastal irrigated ecosystem. Lodging is permanent displacement of vertical stem to leaning horizontally at reproductive phase. It reduces the yield up to $2 \%$ for every $1 \%$ of lodging (Setter et al., 1997). Besides yield reduction, lodging of rice crop deteriorates quality and impedes manual as well as mechanical harvesting and also increases drying costs. Direct evaluation of lodging resistance requires visual estimates in plots of advanced breeding lines or cultivars. A true assessment needs simulating conditions favorable to lodging, with a large investment of time, money and resources. But even routine plot scoring cannot be used to evaluate first generations in breeding programs, because their genetic variability is too high to grow plots. Indirect selection for lodging resistance related traits is one of the strategies to develop non lodging rice cultivars. Breaking resistance and lodging index of lower nodes were significantly correlated with visual score of lodging (Islam et al., 2007). Zhang et al., (2009) reported that selection of lodging 
resistant cultivars, with thicker culm wall, larger culm wall area, better vascular bundle characters with high cellulose content in their stem would be useful. Wu et al., (2011) reported that the large culm cultivars exhibited greater plant size, culm diameter and flag leaf length and width, as well as lower tiller numbers. Pedigree analysis showed lodging resistance of varieties was closely correlated with that of their parents (Ming-liang et al., 2012). Yang et al., (2012) reported that breaking resistance strengths of three elongation internodes near stem base were significantly correlated with internode wall thickness and panicle length was significantly positively correlated with internode wall thickness. Laza et al., (2014) reported positive correlations of percentage lodging with canopy height, culm length and negative correlation with stem diameter.

Indirect selection through lodging resistant related traits like culm diameter, culm thickness, culm strength, bending strength, basal internodal length, per cent of lodging in early generations is a herculean task as it involves handling of large population. Several workers reported QTLS for culm diameter (Ookawa et al., 2010), culm thickness (Mu et al., 2004), culm strength (Zhu et al., 2008; Yamamoto et al., 2013; Yano et al., 2014), basal internodal length (Mu et al., 2004) will be useful in selection of lodging resistant lines in early generations. Hence present study aimed to screen $\mathrm{F}_{3}$ families for lodging resistance using molecular markers and phenotypic screening.

\section{Materials and Methods}

One hundred eighty $\mathrm{F}_{3}$ families derived from lodging susceptible parent Swarna and resistant elite lines II 110-9-1-1-1-1 and 160 from Swarna and resistant line MTU 1121 were evaluated in 2 replications along with checks during Rabi 2013-14. Each $\mathrm{F}_{3}$ family was planted in 2 rows per replication with row length of $3 \mathrm{~m}$ with spacing of 20 X15 cm.

Data pertaining to 14 phenotypic characters phenotypic characters viz., days to $50 \%$ flowering, plant height, number of ear bearing tillers plant ${ }^{-1}$, panicle length, number of filled grains panicle ${ }^{-1}$, spikelet fertility, test weight, grain yield plant $^{-1}$, culm diameter, culm thickness, culm strength, $4^{\text {th }}$ internodal length, bending strength and per cent of lodging were collected among $\mathrm{F}_{3}$ families. Lodging resistance traits, culm diameter and culm thickness was measured at $4^{\text {th }}$ internodal length from the top using vernier calipers at 20 days after heading, culm strength and per cent of lodging was measured as per the IRRI standard evaluation systems, 2002. Bending strength was measured by pushing hill at $20 \mathrm{~cm}$ above the ground at $45^{\circ}$ angle using Prostrate tester (DIK 7401, Daiki Rika Kogyo Co. Ltd., Tokyo, Japan and it was expressed in $\mathrm{g} /$ stem using the following formula as per Bhagat et al., (2011).

Bending strength $=[$ (test reading/40) $\mathrm{X}$ (1000/number of tillers)]

$\mathrm{F}_{3}$ families exhibiting 0 to 25 per cent of lodging was considered as resistant families and families showing per cent of lodging more than 25 per cent were grouped as lodging susceptible.

\section{Genotypic screening with molecular markers}

DNA was isolated from the leaf samples of $\mathrm{F}_{3}$ families collected at 45 days using the method described by Zheng et al., (1995). Microsatellite markers RM 20557, RM 5509 associated with Strong culm 2 (SCM2) (Ookawa et al., 2010) confers for lodging resistance with wider culms on chromosome 6 showing polymorphism between parents were used to screen $F_{3}$ families derived from 
Swarna and II 110-9-1-1-1-1. Whereas RM 6933 on chromosome 2 and RM 216 on chromosome 10 associated with culm strength (Yano et al., 2014 and $\mathrm{Mu}$ et al., 2004) exhibiting polymorphism between parents Swarna and MTU 1121 were used to screen respective $\mathrm{F}_{3}$ families.

PCR was performed using 10ul reaction mixture comprising of $2.5 \mathrm{ul}$ of $20 \mathrm{ng}$ of genomic DNA, $1 \mu \mathrm{l}$ of $10 \mathrm{pmol}$ primer of forward and reverse each, $0.5 \mu \mathrm{l}$ of $2.5 \mathrm{mM}$ deoxy ribonucleotides (dNTPS), $1 \mu \mathrm{l}$ of Genei $10 \mathrm{X}$ assay buffer $\left(10 \mathrm{mM}\right.$ TrisHCl $\left(\mathrm{p}^{\mathrm{H}} 8.3\right)$, $50 \mathrm{mM} \mathrm{KCl}, 1.5 \mathrm{mM} \mathrm{MgCl} 2,0.01 \%$ gelatin) and $1 \mu \mathrm{l}$ of $1 \mathrm{U} / \mu \mathrm{l}$ Taq DNA polymerase (Genei ${ }^{\circledR}$ ) and $3 \mu \mathrm{l}$ of sterile distilled.

Thermo profile of $94^{\circ} \mathrm{C}$ for 5 minutes for initial, 30 seconds for denaturation, annealing temperature at $58^{\circ} \mathrm{C}$ for 30 seconds, elongation at $72^{\circ} \mathrm{C}$ for 1 minute and final extension with same temperature for 1 minute was followed for Eppendorf thermocylcer for amplification of the products. The PCR products were subjected to electrophoresis on $3 \%$ high resolution agarose gel unit containing $1 \mathrm{X}$ TAE buffer. The DNA fragments were captured using gel documentation system (SYNGENE Gene flash U.K.).

\section{Data analysis of $F_{3}$ families}

Clustering of $180 \mathrm{~F}_{3}$ families derived from MTU 7029/II 110-9-1-1-1-1 cross combination and $160 \mathrm{~F}_{3}$ families derived from MTU 7029/MTU 1121 was done using 14 characters by Mahalonobis $\mathrm{D}^{2}$ Ward's Minimum Variance method. Graphical genotyping of $\mathrm{F}_{3}$ families was performed using GGT 2.0 version (Van Berloo 1999). Correlation coefficients were estimated for paired character association of 14 characters among the two crosses of $F_{3}$ families as per Falconer, 1964.

\section{Results and Discussion}

Identification of lodging resistant $f_{3}$ families using molecular markers

Marker RM 20557 segregated among $180 \mathrm{~F}_{3}$ families of MTU 7029/II 110-9-1-1-1-1 as $51.1 \%$ for lodging susceptible parent MTU $7029,35 \%$ of donor allele of resistant parent II 110-9-1-1-1 and 13.3\% heterozygous allelic pattern (Table 1). RM 5509 segregated as $36.7 \%$ for lodging susceptible parent MTU $7029,51.7 \%$ donor allele of II 110-9-1-1-1-1 and $8.9 \%$ of heterozygote allele. It indicated that both the markers expressed fixation of alleles towards homozygosity of either parents in $\mathrm{F}_{3}$ generation. These two markers with homozygosity allele of donor and heterozygote allele can be used to select lodging resistant families in early generation of cross MTU 7029/II 110-9-1-1-1-1.

In case of $\mathrm{F}_{3}$ families derived from MTU 7029/MTU 1121 cross combination, RM 6933 segregated among 160 families as $41.9 \%$ towards susceptible parent MTU 7029 and $24.4 \%$ for donor allele of MTU 1121 and 21.3 $\%$ heterozygous allele. Another marker RM 216 showed segregation pattern of $23.8 \%$ for susceptible parent allele, $42.5 \%$ donor parent and $31.9 \%$ heterozygous allele. RM 6933 fixed homozygosity more towards susceptible parent and RM 216 towards resistant parent. Approximately $20-30 \%$ of $\mathrm{F}_{3}$ families expressed heterozygous allele for both the markers. Selection of lodging resistant loci using these two markers for donor allele and heterozygous allele is useful in early generations.

\section{Identification of lodging resistant lines using phenotypic characters}

Analysis of variance revealed significant differences among the $\mathrm{F}_{3}$ families of both the cross combinations for yield and lodging 
related characters. The results of Mahalanobis $\mathrm{D}^{2}$ revealed that $180 \quad \mathrm{~F}_{3}$ families of MTU 7029/ II 110-9-1-1-1-1 were grouped into 14 clusters (Table 2). Most of the lodging resistant $\mathrm{F}_{3}$ families were grouped in first five clusters. Cluster 14 has the highest intra cluster value of 68.25 . Inter cluster mean values were higher between clusters 6 and 14 followed by clusters 1 and 14 . Cluster 4 had the all lodging resistant lines. Inter cluster values were higher between clusters 6 and 14 (118.73) followed by 1 and 14 clusters (101.55) and the results were depicted in the Table $3 . \mathrm{F}_{3}$ families with medium duration were grouped in cluster 10 (100.65 days). Clusters13 has $\mathrm{F}_{3}$ families with intermediate height $(126.88 \mathrm{~cm})$.

More number of ear bearing tillers per plant of 27.92 were observed in cluster 14. Clusters 9, 10, 1 and 4 had the families with long panicles. $\mathrm{F}_{3}$ lines in clusters 1,5 and 8 expressed higher spikelet fertility per cent of $88.47,88.20$ and 88.07 respectively. Cluster 4 with more number of filled grains per panicle (237.26) and the cluster 1 consist of $F_{3}$ families with highest test weight (21.56 g). Higher grain yield plant ${ }^{-1}$ was observed in the cluster $14(33.92 \mathrm{~g})$ followed by cluster 4 (32.12 g).

$\mathrm{F}_{3}$ families with wider culm $(7.45 \mathrm{~mm})$ were grouped in cluster 9. Thicker culms were observed in cluster 12 with mean value of 1.47 $\mathrm{mm} . \mathrm{F}_{3}$ families with strong culm (1.36) and lowest per cent of lodging (0.38) were grouped in cluster 4 . Highest bending strength was observed in cluster $2\left(51.91 \mathrm{~g} \mathrm{stem}^{-1}\right)$. Cluster 12 possessed the genotypes with shortest basal inter nodal length of $6.76 \mathrm{~cm}$ (Table 4). Thus, lodging resistant genotypes with high yield, strong and wider culms were grouped in clusters 4 and 14 and these families can be further advanced to next generation for the development of non-lodging high yielding varieties.
One hundred sixty $F_{3}$ families derived from MTU 7029/MTU 1121 were grouped into 13 clusters (Table 5). In this cross combination, 150 families were found to be lodging resistant. Lodging susceptible genotypes were fallen under clusters 12, 13 and 9. Highest inter cluster value of 95.26 was observed between clusters 6 and 13 (Table 6).

Lodging resistant lines with shortest duration were grouped in cluster 2 (99.71) and late duration in cluster 12 (111.25) and details are presented in Table 7. Lodging resistant lines with semi dwarf stature were grouped in cluster 5 with mean value of $103.50 \mathrm{~cm}$. More number of ear bearing tillers plant $^{-1}$ were observed in cluster 4 (16.88).

Lodging resistant lines with long panicles were together formed into cluster 11 (28.23). More number of filled grains panicle ${ }^{-1}$ was observed in cluster 3 (257.16). Lodging resistant lines with higher spikelet fertility were found in cluster $6(89.21 \%)$. Lodging resistant lines with bold grains were observed in cluster $7(22.40 \mathrm{~g})$.

Lodging resistant lines with higher grain yield plant $^{-1}$ were found in cluster 4 (23.29 g). $F_{3}$ families with wider and thicker culms were grouped in cluster 8. Lines with short basal inter nodal length were grouped in cluster 9 $(4.78 \mathrm{~cm})$. Higher cluster mean value for bending strength was observed in cluster 6 $\left(99.68 \mathrm{~g} \mathrm{stem}^{-1}\right)$ and minimal per cent of lodging was observed in cluster $2(0.90 \%)$. Thus, eight lodging resistant $\mathrm{F}_{3}$ lines with higher yield were grouped in cluster 4 followed by 14 lodging resistant lines in cluster 8 can be advanced to next generation for development of high yielding non lodging varieties.

But generation of data for large population in early generation in regular plant breeding programmes is herculean task. 
Table.1 Performance of lodging resistant loci linked SSR markers over $\mathrm{F}_{3}$ families in two crosses

\begin{tabular}{|l|l|c|l|l|l|l|l|}
\hline Cross & Marker & Chromosome & $\begin{array}{l}\text { Position } \\
\text { Mb }\end{array}$ & $\begin{array}{l}\text { Missing } \\
\text { allele } \\
(\boldsymbol{\%})\end{array}$ & A (\%) & B (\%) & H (\%) \\
\hline $\begin{array}{l}\text { MTU7029/ } \\
\text { II110-9-1-1-1 }\end{array}$ & RM20557 & 6 & 27.50 & 0.60 & 51.10 & 35.00 & 13.30 \\
\hline $\begin{array}{l}\text { MTU7029/ } \\
\text { II110-9-1-1-1 }\end{array}$ & RM5509 & 6 & 27.80 & 1.10 & 36.70 & 51.70 & 8.90 \\
\hline $\begin{array}{l}\text { MTU 7029 / } \\
\text { MTU1121 }\end{array}$ & RM6933 & 2 & 29.30 & 12.40 & 41.90 & 24.40 & 21.30 \\
\hline $\begin{array}{l}\text { MTU 7029/ } \\
\text { MTU1121 }\end{array}$ & RM216 & 10 & 5.30 & 1.80 & 23.80 & 42.50 & 31.90 \\
\hline
\end{tabular}

A: Susceptible parent allele, B: Resistant parent allele H: Heterozygous allele

Table.2 Clustering pattern $\mathrm{F}_{3}$ families pertaining to cross combination MTU 7029/II 110-9-1-1-1 during rabi 2013-14

\begin{tabular}{|c|c|c|c|}
\hline $\begin{array}{l}\text { Cluster } \\
\text { number }\end{array}$ & $\begin{array}{l}\text { Number } \\
\text { of } F_{3} \text { lines } \\
\text { in cluster }\end{array}$ & $\begin{array}{l}\text { Number of } \\
\text { lodging resistant } \\
F_{3} \text { lines }\end{array}$ & Details of lodging resistant $F_{3}$ lines \\
\hline 1 & 16 & 11 & $\begin{array}{l}\text { LH12-1, LH12-13, LH12-97, LH12-47, LH12-127, LH12- } \\
\text { 8, LH12-26, LH12-25, LH12-58, LH12-96, LH12-28 }\end{array}$ \\
\hline 2 & 27 & 18 & $\begin{array}{l}\text { LH12-27, LH12-142, LH12-29, LH12-9, LH12-11, LH12- } \\
\text { 66, LH12-65, LH12-9, LH12-122, LH12-116, LH12-129, } \\
\text { LH12-130, LH12-132, LH12-124, LH12-141, LH12-121, } \\
\text { LH12-154, LH12-89 }\end{array}$ \\
\hline 3 & 15 & 9 & $\begin{array}{l}\text { LH12-24, LH12-114, LH12-88, LH12-123, LH12-94, } \\
\text { LH12-94, LH1-95, LH12-87, LH12-151 }\end{array}$ \\
\hline 4 & 14 & 14 & $\begin{array}{l}\text { LH12-96, LH12-105, LH12-92, LH12-111, LH12-99, } \\
\text { LH12-91, LH12-93, LH12-147, LH12-101, LH12-106, } \\
\text { LH12-38, LH12-109, LH12-144, LH12-115 }\end{array}$ \\
\hline 5 & 10 & 9 & $\begin{array}{l}\text { LH12-45, LH12-150, LH12-113, LH12-137, LH12-15, } \\
\text { LH12-123, LH12-140, LH12-136, LH12-140 }\end{array}$ \\
\hline 6 & 1 & 0 & 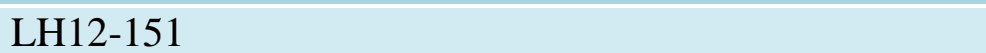 \\
\hline 7 & 26 & 0 & 0 \\
\hline 8 & 10 & 0 & 0 \\
\hline 9 & 10 & 1 & LH12-181 \\
\hline 10 & 10 & 2 & LH12-64, LH12-120 \\
\hline 11 & 10 & 0 & 0 \\
\hline 12 & 14 & 0 & 0 \\
\hline 13 & 15 & 0 & 0 \\
\hline 14 & 2 & 1 & LH12-118 \\
\hline Total & 180 & 65 & \\
\hline
\end{tabular}


Table.3 Intra and inter cluster means of $\mathrm{F}_{3}$ families pertaining to cross combination MTU 7029/II 110-9-1-1-1-1 during rabi 2013-14

\begin{tabular}{|c|c|c|c|c|c|c|c|c|c|c|c|c|c|c|}
\hline Clusters & $\begin{array}{l}\text { Cluster } \\
1\end{array}$ & $\begin{array}{l}\text { Cluster } \\
2\end{array}$ & $\begin{array}{l}\text { Cluster } \\
3\end{array}$ & $\begin{array}{l}\text { Cluster } \\
4\end{array}$ & $\begin{array}{l}\text { Cluster } \\
5\end{array}$ & $\begin{array}{l}\text { Cluster } \\
6\end{array}$ & $\begin{array}{l}\text { Cluster } \\
7\end{array}$ & $\begin{array}{l}\text { Cluster } \\
8\end{array}$ & $\begin{array}{l}\text { Cluster } \\
9\end{array}$ & $\begin{array}{l}\text { Cluster } \\
10\end{array}$ & $\begin{array}{l}\text { Cluster } \\
11\end{array}$ & $\begin{array}{l}\text { Cluster } \\
12\end{array}$ & Cluster13 & $\begin{array}{l}\text { Cluster } \\
14\end{array}$ \\
\hline Cluster1 & 19.52 & 22.62 & 23.08 & 27.11 & 28.42 & 85.92 & 29.19 & 34.41 & 34.12 & 35.76 & 31.83 & 34.59 & 33.41 & 101.55 \\
\hline Cluster2 & & 13.23 & 18.37 & 20.64 & 20.48 & 70.11 & 22.21 & 31.60 & 28.02 & 28.07 & 28.13 & 27.56 & 24.52 & 96.79 \\
\hline Cluster3 & & & 14.71 & 22.12 & 20.62 & 60.72 & 27.35 & 32.29 & 31.46 & 29.02 & 26.68 & 31.54 & 25.32 & 85.86 \\
\hline Cluster4 & & & & 13.75 & 24.44 & 83.50 & 28.19 & 39.43 & 33.84 & 33.00 & 39.03 & 38.15 & 36.84 & 83.04 \\
\hline Cluster5 & & & & & 13.19 & 48.29 & 29.63 & 35.12 & 42.37 & 37.87 & 38.30 & 37.04 & 31.01 & 82.78 \\
\hline Cluster6 & & & & & & 0.00 & 67.61 & 55.75 & 75.05 & 72.86 & 63.16 & 73.71 & 62.39 & 118.73 \\
\hline Cluster7 & & & & & & & 13.28 & 19.28 & 19.74 & 24.83 & 24.20 & 20.20 & 22.02 & 87.42 \\
\hline Cluster8 & & & & & & & & 17.10 & 25.40 & 28.12 & 25.05 & 23.48 & 28.81 & 86.92 \\
\hline Cluster9 & & & & & & & & & 14.96 & 27.57 & 26.55 & 25.88 & 24.73 & 99.39 \\
\hline$\overline{\text { Cluster10 }}$ & & & & & & & & & & 22.63 & 28.97 & 29.54 & 33.80 & 77.55 \\
\hline Cluster11 & & & & & & & & & & & 16.36 & 21.90 & 24.30 & 100.90 \\
\hline Cluster12 & & & & & & & & & & & & 15.93 & 22.90 & 88.18 \\
\hline Cluster13 & & & & & & & & & & & & & 17.75 & 98.68 \\
\hline Cluster14 & & & & & & & & & & & & & & 68.25 \\
\hline
\end{tabular}

Table.4 Cluster mean values of 14 characters pertaining to $\mathrm{F}_{3}$ population of MTU 7029/II 110-9-1-1-1-1 during rabi 2013-14

\begin{tabular}{|c|c|c|c|c|c|c|c|c|c|c|c|c|c|c|}
\hline Clusters & $\begin{array}{l}\text { Days to } \\
50 \% \\
\text { flowering }\end{array}$ & $\begin{array}{l}\text { Plant } \\
\text { height } \\
(\mathrm{cm})\end{array}$ & $\begin{array}{l}\text { No. of ear } \\
\text { bearing } \\
\text { tillers } \\
\text { plant-1 }\end{array}$ & $\begin{array}{l}\text { Panicle } \\
\text { length } \\
(\mathrm{cm})\end{array}$ & $\begin{array}{l}\text { No. of } \\
\text { filled } \\
\text { grains } \\
\text { panicle }^{-1}\end{array}$ & $\begin{array}{l}\text { Spikelet } \\
\text { fertility } \\
(\%)\end{array}$ & $\begin{array}{l}\text { Test } \\
\text { weight } \\
\text { (g) }\end{array}$ & $\begin{array}{l}\text { Grain } \\
\text { yield } \\
\text { plant }^{-1} \\
\text { (g) }\end{array}$ & $\begin{array}{l}\text { Culm } \\
\text { diameter } \\
(\mathbf{m m})\end{array}$ & $\begin{array}{l}\text { Culm } \\
\text { thickness } \\
(\mathrm{mm})\end{array}$ & $\begin{array}{l}\text { Culm } \\
\text { strength }\end{array}$ & $\begin{array}{l}4^{\text {th }} \text { Inter } \\
\text { nodal } \\
\text { length } \\
(\mathrm{cm})\end{array}$ & $\begin{array}{l}\text { Bending } \\
\text { Strength } \\
\left(\mathrm{g} \mathrm{stem}^{-1}\right)\end{array}$ & $\begin{array}{l}\text { Lodging } \\
\text { (\%) }\end{array}$ \\
\hline Cluster1 & 106.47 & 134.67 & 10.01 & 28.47 & 146.35 & 88.47 & 21.56 & 22.21 & 7.14 & 1.01 & 2.96 & 7.26 & 49.30 & 12.90 \\
\hline Cluster2 & 107.65 & 135.58 & 9.17 & 26.30 & 182.67 & 80.47 & 15.41 & 20.21 & 7.03 & 1.24 & 3.17 & 7.76 & 51.91 & 19.02 \\
\hline Cluster3 & 109.27 & 133.38 & 11.04 & 27.15 & 135.21 & 70.20 & 17.18 & 22.43 & 6.41 & 0.88 & 2.20 & 8.14 & 45.61 & 16.92 \\
\hline Cluster 4 & 111.14 & 133.26 & 11.60 & 28.41 & 237.26 & 82.37 & 16.31 & 32.12 & 6.60 & 1.11 & 1.36 & 7.62 & 45.52 & 0.38 \\
\hline Cluster5 & 108.15 & 130.50 & 10.30 & 24.25 & 169.22 & 88.20 & 16.53 & 21.72 & 5.60 & 1.06 & 1.87 & 10.79 & 48.83 & 4.93 \\
\hline Cluster7 & 107.29 & 139.01 & 9.97 & 27.00 & 220.07 & 85.99 & 17.07 & 20.38 & 6.66 & 1.14 & 7.65 & 7.56 & 21.28 & 78.71 \\
\hline Cluster8 & 106.60 & 152.38 & 10.07 & 27.74 & 156.37 & 88.07 & 17.89 & 21.65 & 6.56 & 0.90 & 8.53 & 9.48 & 15.69 & 90.70 \\
\hline Cluster 9 & 111.50 & 134.97 & 10.38 & 32.12 & 180.85 & 80.31 & 15.69 & 18.79 & 7.45 & 1.21 & 8.40 & 7.45 & 17.63 & 77.80 \\
\hline Cluster10 & 100.65 & 143.65 & 12.52 & 29.39 & 173.09 & 71.63 & 14.71 & 24.62 & 6.94 & 1.14 & 5.93 & 7.96 & 23.13 & 55.76 \\
\hline Cluster11 & 108.00 & 140.87 & 9.15 & 26.22 & 113.92 & 63.99 & 19.64 & 24.00 & 7.29 & 1.22 & 7.47 & 8.22 & 24.98 & 87.17 \\
\hline Cluster12 & 109.36 & 146.85 & 11.49 & 24.40 & 133.60 & 83.16 & 17.23 & 20.93 & 7.00 & 1.47 & 7.71 & 6.76 & 17.22 & 83.34 \\
\hline Cluster13 & 111.90 & 126.88 & 9.83 & 25.29 & 130.33 & 77.35 & 15.92 & 15.11 & 6.63 & 1.13 & 6.88 & 7.19 & 27.25 & 79.52 \\
\hline Cluster14 & 102.75 & 137.00 & 27.92 & 24.43 & 187.67 & 86.03 & 14.46 & 33.92 & 5.75 & 0.86 & 5.00 & 11.58 & 13.89 & 49.00 \\
\hline
\end{tabular}


Table.5 Clustering pattern $\mathrm{F}_{3}$ lines pertaining to cross combination MTU 7029/MTU1121 during rabi 2013-14

\begin{tabular}{|c|c|c|c|}
\hline $\begin{array}{l}\text { Cluster } \\
\text { number }\end{array}$ & $\begin{array}{l}\text { Number } \\
\text { of } F_{3} \text { lines } \\
\text { in cluster }\end{array}$ & $\begin{array}{l}\text { Number of } \\
\text { lodging } \\
\text { resistant } \mathrm{F}_{3} \\
\text { lines }\end{array}$ & Details of lodging resistant $F_{3}$ lines \\
\hline Cluster 1 & 27 & 27 & $\begin{array}{l}\text { LH14-1, LH14-306, LH14-71, LH14-167, LH14-179, } \\
\text { LH14-308, LH14-23, LH14-314, LH14-170, LH14- } \\
\text { 189, LH14-315, LH14-11, LH14-93, LH14-118, LH14- } \\
\text { 188, LH14-28, LH14-35, LH14-80, LH14-12, LH14- } \\
\text { 310, LH14-166, LH14-56, LH14-108, LH14-121, } \\
\text { LH14-311, LH14-183, LH14-195 }\end{array}$ \\
\hline Cluster 2 & 14 & 14 & $\begin{array}{l}\text { LH14-3, LH14-169, LH14-9, LH14-75, LH14-147, } \\
\text { LH14-16, LH14-151, LH14-155, LH14-156, LH14- } \\
\text { 150, LH14-163, LH14-89, LH14-165, LH14-187 }\end{array}$ \\
\hline Cluster 3 & 10 & 10 & $\begin{array}{l}\text { LH14-24, LH14-49, LH14-159, LH14-161, LH14-185 } \\
\text { LH14-54, LH14-76, LH14-30, LH14-146, LH14-41 }\end{array}$ \\
\hline Cluster 4 & 8 & 8 & $\begin{array}{l}\text { LH14-160, LH14-301, LH14-317, LH14-176, LH14- } \\
\text { 199, LH14-309, LH14-313, LH14-198 }\end{array}$ \\
\hline Cluster 5 & 20 & 20 & $\begin{array}{l}\text { LH14-2, LH14-95, LH14-126, LH14-184, LH14-25 } \\
\text { LH14-149, LH14-97, LH14-307, LH14-302, LH14- } \\
\text { 312, LH14-318, LH14-32, LH14-120, LH14-77, LH14- } \\
\text { 78, LH14-69, LH14-88, LH14-125, LH14-81, LH14-68 }\end{array}$ \\
\hline Cluster 6 & 2 & 2 & LH14-91, LH14-324 \\
\hline Cluster 7 & 18 & 18 & $\begin{array}{l}\text { LH14-4, LH14-37, LH14-39, LH14-26, LH14-27, } \\
\text { LH14-102, LH14-113, LH14-137, LH14-18, LH14- } \\
\text { 130, LH14-131, LH14-31, LH14-79, LH14-86, LH14- } \\
\text { 104, LH14-174, LH14-175, LH14-124 }\end{array}$ \\
\hline Cluster 8 & 14 & 14 & $\begin{array}{l}\text { LH14-6, LH14-111, LH14-114, LH14-55, LH14-123 } \\
\text { LH14-21, LH14-323, LH14-38, LH14-116, LH14-40 } \\
\text { LH14-52, LH14-51, LH14-67, LH14-320 }\end{array}$ \\
\hline Cluster 9 & 12 & 11 & $\begin{array}{l}\text { LH14-5, LH14-14, LH14-180, LH14-29, LH14-322, } \\
\text { LH14-10, LH14-43, LH14-48, LH14-61, LH14-46, } \\
\text { LH14-64, LH14-158 }\end{array}$ \\
\hline Cluster 10 & 15 & 15 & $\begin{array}{l}\text { LH14-42, LH14-148, LH14-63, LH14-164, LH14-100, } \\
\text { LH14-98, LH14-128, LH14-87, LH14-70, LH14-129, } \\
\text { LH14-90, LH14-96, LH14-105, LH14-110, LH14-319 }\end{array}$ \\
\hline Cluster 11 & 7 & 7 & $\begin{array}{l}\text { LH14-72, LH14-303, LH14-34, LH14-141, LH14-171, } \\
\text { LH14-316, LH14-103 }\end{array}$ \\
\hline Cluster 12 & 9 & 4 & L LH14-73, LH14-82, LH14-66, LH14-53 \\
\hline Cluster 13 & 4 & 0 & 0 \\
\hline Total & 160 & 150 & \\
\hline
\end{tabular}


Table.6 Intra and inter cluster means of $\mathrm{F}_{3}$ families pertaining to cross combination MTU 7029/MTU 1121 during rabi $2013-14$

\begin{tabular}{|c|c|c|c|c|c|c|c|c|c|c|c|c|c|}
\hline Clusters & $\begin{array}{l}\text { Cluster } \\
1\end{array}$ & $\begin{array}{l}\text { Cluster } \\
2\end{array}$ & $\begin{array}{l}\text { Cluster } \\
\mathbf{3}\end{array}$ & $\begin{array}{l}\text { Cluster } \\
4\end{array}$ & $\begin{array}{l}\text { Cluster } \\
\mathbf{5}\end{array}$ & $\begin{array}{l}\text { Cluster } \\
6\end{array}$ & $\begin{array}{l}\text { Cluster } \\
7\end{array}$ & $\begin{array}{l}\text { Cluster } \\
\mathbf{8}\end{array}$ & $\begin{array}{l}\text { Cluster } \\
9\end{array}$ & $\begin{array}{l}\text { Cluster } \\
10\end{array}$ & $\begin{array}{l}\text { Cluster } \\
11\end{array}$ & $\begin{array}{l}\text { Cluster } \\
12\end{array}$ & $\begin{array}{l}\text { Cluster } \\
13\end{array}$ \\
\hline Cluster 1 & 14.77 & 18.83 & 19.84 & 22.61 & 21.19 & 56.47 & 24.51 & 21.98 & 20.42 & 21.21 & 37.51 & 32.42 & 55.73 \\
\hline Cluster 2 & & 13.90 & 21.37 & 34.20 & 21.22 & 49.56 & 26.56 & 22.51 & 24.07 & 23.39 & 42.21 & 30.96 & 62.57 \\
\hline Cluster 3 & & & 12.65 & 25.32 & 24.48 & 54.01 & 27.61 & 19.24 & 24.09 & 26.59 & 50.46 & 39.26 & 52.12 \\
\hline Cluster 4 & & & & 16.75 & 37.68 & 79.55 & 30.71 & 27.31 & 27.20 & 33.47 & 45.52 & 46.73 & 57.03 \\
\hline Cluster 5 & & & & & 15.06 & 40.48 & 25.27 & 24.92 & 29.88 & 23.55 & 48.28 & 34.83 & 69.24 \\
\hline Cluster 6 & & & & & & 37.65 & 46.36 & 40.75 & 58.86 & 43.08 & 83.46 & 54.13 & 95.26 \\
\hline Cluster 7 & & & & & & & 20.43 & 20.30 & 27.67 & 24.72 & 45.24 & 35.39 & 61.84 \\
\hline Cluster 8 & & & & & & & & 10.63 & 21.05 & 20.95 & 43.01 & 37.99 & 56.20 \\
\hline Cluster 9 & & & & & & & & & 16.36 & 21.87 & 34.21 & 31.12 & 43.87 \\
\hline Cluster10 & & & & & & & & & & 15.79 & 34.34 & 28.12 & 58.52 \\
\hline Cluster11 & & & & & & & & & & & 35.62 & 43.83 & 73.73 \\
\hline Cluster12 & & & & & & & & & & & & 24.61 & 45.81 \\
\hline Cluster13 & & & & & & & & & & & & & 16.66 \\
\hline
\end{tabular}

Table.7 Cluster mean values of 14 characters pertaining to $F_{3}$ population of MTU 7029/MTU 1121 during rabi 2013-14

\begin{tabular}{|c|c|c|c|c|c|c|c|c|c|c|c|c|c|c|}
\hline Clusters & $\begin{array}{l}\text { Days to } \\
50 \% \\
\text { flowering }\end{array}$ & $\begin{array}{l}\text { Plant } \\
\text { height } \\
(\mathrm{cm})\end{array}$ & $\begin{array}{l}\text { No. of ear } \\
\text { bearing } \\
\text { tillers } \\
\text { plant-1 }\end{array}$ & $\begin{array}{l}\text { Panicle } \\
\text { length } \\
(\mathrm{cm})\end{array}$ & $\begin{array}{l}\text { No. of } \\
\text { filled } \\
\text { grains } \\
\text { panicle }^{-1}\end{array}$ & $\begin{array}{l}\text { Spikelet } \\
\text { fertility } \\
(\%)\end{array}$ & $\begin{array}{l}\text { Test } \\
\text { weight (g) }\end{array}$ & $\begin{array}{l}\text { Grain } \\
\text { yield } \\
\text { plant }^{-1} \\
(\mathrm{~g})\end{array}$ & $\begin{array}{l}\text { Culm } \\
\text { diameter } \\
(\mathbf{m m})\end{array}$ & $\begin{array}{l}\text { Culm } \\
\text { thickness } \\
(\mathrm{mm})\end{array}$ & $\begin{array}{l}\text { Culm } \\
\text { strength }\end{array}$ & $\begin{array}{l}\text { 4th Inter } \\
\text { nodal } \\
\text { length } \\
(\mathrm{cm})\end{array}$ & $\begin{array}{l}\text { Bending } \\
\text { Strength } \\
\text { (g stem-1) }\end{array}$ & $\begin{array}{l}\text { Per cent } \\
\text { of lodging } \\
(\%)\end{array}$ \\
\hline Cluster1 & 105.76 & 106.54 & 12.23 & 25.75 & 159.70 & 84.48 & 18.11 & 20.04 & 6.42 & 1.21 & 2.67 & 5.85 & 40.92 & 1.65 \\
\hline Cluster2 & 99.71 & 105.92 & 9.61 & 25.77 & 138.92 & 83.00 & 17.94 & 17.97 & 6.54 & 1.17 & 4.12 & 5.60 & 51.44 & 0.90 \\
\hline Cluster3 & 104.10 & 105.99 & 10.58 & 26.43 & 257.16 & 86.06 & 18.51 & 19.81 & 7.05 & 1.37 & 4.30 & 5.04 & 44.37 & 5.90 \\
\hline Cluster4 & 106.75 & 107.97 & 16.88 & 27.14 & 193.56 & 83.53 & 20.28 & 23.69 & 7.12 & 1.42 & 3.21 & 5.99 & 28.74 & 1.21 \\
\hline Cluster5 & 105.70 & 103.50 & 8.39 & 25.30 & 180.66 & 84.33 & 19.69 & 20.60 & 6.34 & 0.90 & 1.88 & 6.61 & 61.95 & 2.65 \\
\hline Cluster6 & 107.50 & 124.17 & 5.25 & 24.89 & 177.75 & 89.21 & 20.44 & 14.50 & 6.97 & 1.61 & 3.33 & 7.47 & 99.68 & 14.07 \\
\hline Cluster7 & 105.61 & 117.53 & 10.37 & 27.18 & 158.47 & 81.13 & 22.40 & 18.97 & 6.98 & 1.07 & 2.74 & 6.56 & 48.40 & 1.48 \\
\hline Cluster8 & 104.93 & 116.07 & 9.58 & 28.13 & 172.65 & 83.30 & 19.83 & 21.33 & 7.62 & 1.62 & 2.36 & 5.54 & 53.83 & 5.30 \\
\hline Cluster9 & 108.92 & 109.27 & 11.24 & 27.20 & 123.88 & 74.29 & 18.16 & 20.49 & 6.92 & 1.40 & 4.75 & 4.78 & 42.29 & 10.30 \\
\hline Cluster10 & 108.87 & 107.37 & 9.80 & 25.84 & 129.00 & 77.01 & 18.72 & 13.95 & 7.22 & 1.39 & 2.38 & 6.34 & 51.52 & 2.59 \\
\hline Cluster11 & 107.79 & 105.22 & 13.00 & 28.23 & 83.89 & 51.62 & 17.52 & 15.13 & 6.55 & 1.43 & 1.81 & 6.16 & 39.62 & 1.59 \\
\hline Cluster12 & 106.72 & 109.42 & 9.70 & 25.11 & 111.07 & 69.25 & 18.70 & 13.76 & 6.64 & 1.10 & 6.33 & 7.45 & 46.50 & 32.44 \\
\hline Cluster13 & 111.25 & 111.92 & 11.33 & 27.92 & 170.00 & 78.72 & 18.53 & 18.58 & 7.07 & 1.26 & 8.17 & 5.18 & 23.53 & 97.13 \\
\hline
\end{tabular}


Table.8 Correlation coefficients of $\mathrm{F}_{3}$ population of MTU 7029/II 110-9-1-1-1-1 during rabi 2013-14

\begin{tabular}{|c|c|c|c|c|c|c|c|c|c|c|c|c|c|c|}
\hline Character & $\begin{array}{l}\text { Days to } \\
50 \% \\
\text { flowerin } \\
\mathrm{g}\end{array}$ & $\begin{array}{l}\text { Plant } \\
\text { height } \\
\text { (cm) }\end{array}$ & $\begin{array}{l}\text { No. of } \\
\text { ear } \\
\text { bearing } \\
\text { tillers } \\
\text { plant-1 }\end{array}$ & $\begin{array}{l}\text { Panicle } \\
\text { length } \\
\text { (cm) }\end{array}$ & $\begin{array}{l}\text { No. of } \\
\text { filled } \\
\text { grains } \\
\text { panicle } \\
\text { i }\end{array}$ & $\begin{array}{l}\text { Spikele } \\
\text { t } \\
\text { fertility } \\
(\%)\end{array}$ & $\begin{array}{l}\text { Test } \\
\text { weight } \\
\text { (g) }\end{array}$ & $\begin{array}{l}\text { Grain } \\
\text { yield } \\
\text { plant }^{-1} \\
\text { (g) }\end{array}$ & $\begin{array}{l}\text { Culm } \\
\text { diamete } \\
\mathbf{r}(\mathbf{m m})\end{array}$ & $\begin{array}{l}\text { Culm } \\
\text { thicknes } \\
\text { s (mm) }\end{array}$ & $\begin{array}{l}\text { Culm } \\
\text { strength }\end{array}$ & $\begin{array}{l}4^{\text {th }} \text { Inter } \\
\text { nodal } \\
\text { length } \\
(\mathrm{cm})\end{array}$ & $\begin{array}{l}\text { Bending } \\
\text { Strength } \\
\text { (g stem } \\
\text { 1) }\end{array}$ & $\begin{array}{l}\text { Per } \\
\text { cent of } \\
\text { lodging } \\
(\%)\end{array}$ \\
\hline $\begin{array}{l}\text { Days to } 50 \% \\
\text { flowering }\end{array}$ & 1.0000 & -0.0224 & $-0.1217^{*}$ & 0.0321 & 0.0115 & 0.0041 & -0.0201 & 0.0318 & -0.0504 & 0.0560 & -0.0603 & -0.0534 & 0.0651 & 0.0311 \\
\hline Plant height $(\mathrm{cm})$ & & 1.0000 & 0.0364 & $0.1289 *$ & 0.0741 & 0.0122 & $0.1171 *$ & 0.0889 & 0.0967 & 0.1004 & $\begin{array}{l}0.2591 * \\
*\end{array}$ & 0.0454 & $\overline{-}-2411 * *$ & $\begin{array}{l}0.2276 * \\
*\end{array}$ \\
\hline $\begin{array}{l}\text { Number of ear } \\
\text { bearing tillers plant- } \\
1\end{array}$ & & & 1.0000 & 0.0131 & 0.0653 & 0.0067 & $-0.1180^{*}$ & $0.2727 * *$ & -0.0885 & -0.0085 & -0.0999 & 0.0691 & $0.2905 * *$ & -0.0479 \\
\hline Panicle length $(\mathrm{cm})$ & & & & 1.0000 & 0.1859 & 0.0349 & 0.0558 & $0.1226^{*}$ & $0.2565^{* *}$ & -0.0569 & 0.0208 & -0.0820 & -0.0329 & -0.0189 \\
\hline $\begin{array}{l}\text { No. of filled grains } \\
\text { panicle }\end{array}$ & & & & & 1.0000 & $\begin{array}{l}0.2660 * \\
*\end{array}$ & $-\overline{0.1806 * *}$ & $0.2752 * *$ & 0.0085 & $-0.1044^{*}$ & -0.1007 & 0.0033 & 0.0336 & - $0.1317 *$ \\
\hline $\begin{array}{ll}\text { Spikelet } & \text { fertility } \\
(\%) & \end{array}$ & & & & & & 1.0000 & 0.0844 & 0.0982 & -0.0702 & -0.0576 & -0.0510 & -0.0585 & 0.0551 & -0.0666 \\
\hline Test weight (g) & & & & & & & 1.0000 & -0.0313 & $0.1709 * *$ & -0.0457 & 0.0249 & -0.0550 & 0.0416 & 0.0120 \\
\hline $\begin{array}{l}\text { Grain yield plant } \\
\text { (g) }\end{array}$ & & & & & & & & 1.0000 & -0.0052 & $-0.1086^{*}$ & $\begin{array}{l}- \\
0.2826 * \\
*\end{array}$ & 0.0258 & 0.0757 & $\begin{array}{l}- \\
0.2763 * \\
*\end{array}$ \\
\hline $\begin{array}{l}\text { Culm diameter } \\
(\mathrm{mm})\end{array}$ & & & & & & & & & 1.0000 & $0.3098 * *$ & 0.0907 & $-\overline{0.2222 * *}$ & 0.0164 & 0.0739 \\
\hline $\begin{array}{l}\text { Culm thickness } \\
(\mathrm{mm})\end{array}$ & & & & & & & & & & 1.0000 & $\begin{array}{l}0.1442 * \\
*\end{array}$ & $-\overline{0.1682 * *}$ & $-0.1114^{*}$ & 0.1031 \\
\hline Culm strength & & & & & & & & & & & 1.0000 & -0.0889 & - & $0.8231 *$ \\
\hline $\begin{array}{lll}\text { 4th Inter nodal } \\
\text { length }(\mathrm{cm})\end{array}$ & & & & & & & & & & & & 1.0000 & 0.0323 & -0.0859 \\
\hline $\begin{array}{l}\text { Bending Strength } \\
\text { (g stem }^{-1}\end{array}$ & & & & & & & & & & & & & 1.0000 & -0.7247 \\
\hline Per cent of lodging & & & & & & & & & & & & & & 1.0000 \\
\hline
\end{tabular}


Table.9 Correlation coefficients of $\mathrm{F}_{3}$ population of MTU 7029/MTU 1121 during dry season of 2013-14

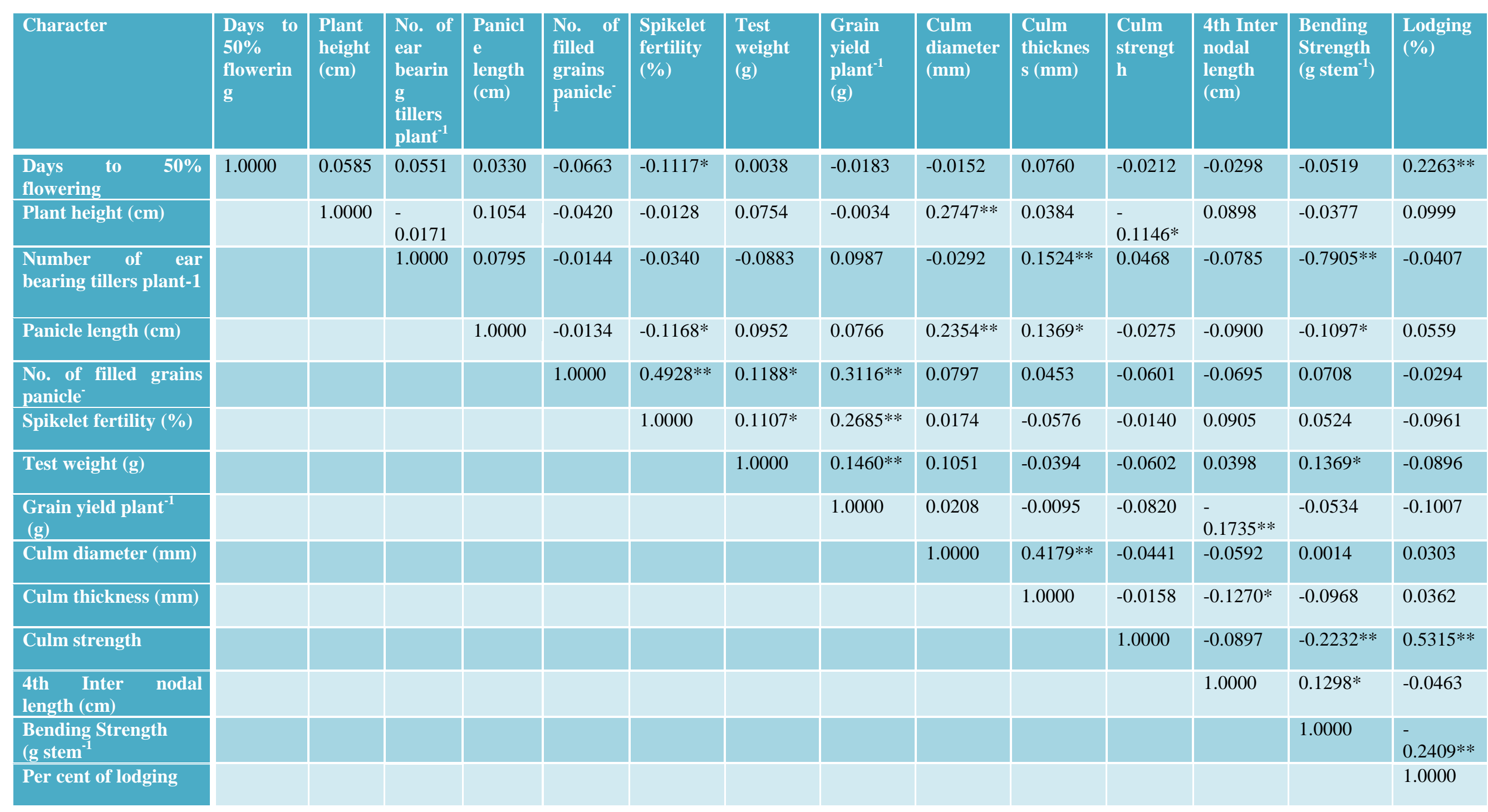


Character association studies of yield and lodging related traits among $F_{3}$ families

Grain yield per plant expressed positive significant association with number of ear bearing tillers plant $^{-1}$, panicle length and number of filled grains per panicle and negatively associated with culm thickness, culm strength and per cent of lodging (Table 8). Per cent of lodging was positively correlated with plant height and culm strength. Per cent of lodging was negatively correlated with grain yield per plant and number of filled grains per panicle. Selection of lodging resistant lines with high yield along with strong culm is desirable in evolving lodging resistant rice varieties from the cross, MTU 7029/II 110-9-1-1-1. Bending strength was negatively correlated with plant height, number of ear bearing tillers per plant, culm thickness and culm strength. Basal inter nodal length was negatively correlated with culm diameter and culm thickness. Culm diameter was positively associated with panicle length and test weight. SCM2 has pleiotropic effect on APO (Apparent panicle organization) as reported by Ookawa et al., (2010). In the present study also culm diameter had positive association with panicle length and donor II 110-9-1-1-1-1 possessed longer panicles with higher yield. Culm strength positively associated with thicker culms. Favourable alleles for lodging resistant lines might be obtained due to allelic interaction of dwarf and strong cum loci. Therefore indirect selection for lodging resistant genotypes with strong culm, minimal per cent of lodging and higher yield is useful in crop improvement. Laza et al., (2014) and Ming-liang et al., (2012) reported positive correlations between percentage lodging and plant height.

Grain yield per plant exhibited significant positive association with number of filled grains per panicle, spikelet fertility $\%$ and test weight, culm thickness and negatively correlated with basal inter nodal length. Per cent of lodging was positively correlated with days to $50 \%$ flowering, culm strength and negatively associated with bending strength among $\mathrm{F}_{3}$ families of MTU 7029/MTU 1121 (Table 9).

In this case, selection of lodging resistant lines with more number of filled grains panicle $^{-1}$ having high spikelet fertility $\%$, test weight and thicker culm with minimal basal inter nodal length is rewarding. Bending strength was negatively correlated with number of ear bearing tillers plant ${ }^{-1}$, panicle length and culm strength and positively associated with test weight and basal inter nodal length. Negative association of SCM3 and SCM4 QTLs conferring for bending strength with number of productive tillers per plant was observed by Yano et al., (2014). Lower elongating internodes have negative association with ear bearing tillers per plant. Basal inter nodal length was negatively associated with grain yield plant ${ }^{-1}$ and culm thickness. Culm strength negatively correlated with plant height. Culm thickness has positive association with number of ear bearing tillers per plant, panicle length and culm diameter. Positive association of culm diameter with plant height and panicle length was observed.

Lodging resistant lines selected though phenotypic characters in both crosses showed co-segregation banding pattern of either homozygous donor allele or heterozygous alleles for the markers used in respective cross indicating usefulness of molecular markers in selection of lodging resistant lines. Selection of lodging resistant lines depends on contribution of resistant alleles from respective donors even though same susceptible parent used. These molecular markers validated in another $F_{3}$ population derived from susceptible parent MTU 1061 and resistant parent BPT 2270, markers 
RM5509 and RM 20557 were showed cosegregation banding pattern for resistant and susceptible families indicating that these markers can be useful in selection of lodging resistant lines in early generations.

Correlation studies revealed that markers RM 20557, RM 5509 are useful in selection of lodging resistant line as positive correlation of culm diameter with panicle length was observed among the $\mathrm{F}_{3}$ families of MTU 7029/II 110-9-1-1-1-1 as in case of linked QTL SCM2 showing similar type of association. Bending strength showed negative association with number of ear bearing tillers per plant was observed in case of $\mathrm{F}_{3}$ families of MTU 7029/MTU 1121 and similar type of association was observed for SCM 3 and SCM4 and marker RM 6933 is in the vicinity of SCM 4 on chromosome 2 . Using above markers associated with lodging resistant related traits would help in selection of lodging resistant lines with homozygosity for donor allele and heterozygous alleles in early generations rather on indirect selection using phenotypic characters of lodging related traits

\section{Acknowledgements}

We acknowledge Acharya N. G Ranga agricultural University for providing opportunity for carrying out above research.

\section{References}

Bhagat, K.P., Sairam, R.K., Deshmukh, P.S and Kushwaha, S.R. 2011. Biochemical analysis of stem in lodging tolerant and susceptible wheat (Triticum aestivum L.) genotypes under normal and late sown conditions. Indian Journal of Journal of Plant Physiology. 16(1): 6874.

Falconer, D.S. 1964. Introduction to Quantitative Genetics. Longmann.
London and New York. Pp. 294-300.

Islam, M.S., Peng, S., Visperas, R.M., Ereful, N., Bhuiya, M.S.U and Julwquar, A.W. 2007. Lodging related morphological traits of hybrid rice in a tropical irrigated ecosystem. Field Crops Research. 101: 240-248.

Laza, M.R., Shrestha, S., Mendez, K.V., Dingkuhn, M and Lopez, M. 2014. Morphological characterization of component traits for lodging resistance in rice. Abstracts of International Rice Congress 2014, October 27 to November1 2014, Bangkok, 1354.

Ming-liang, D., Zhen-x,i A., Qian, Z., Yumin, C., Wei-hua, L and Lu-yuan, D. 2012. Relationship between lodging resistance and either agronomic traits or parents lodging resistance in plateau japonica rice. Chinese Journal of Rice Science. 26(3): 325-330.

Mu, P., Zi-chao, L., Chun-ping, L., Hongliang, Z and Xiang-kun, W. 2004. QTL analysis for lodging resistance in rice using a DH Population under lowland and upland cultural Conditions. Proceedings of the $4^{\text {th }}$ International Crop Science Congress. 26 September 1 October 2004, Brisbane, Australia.

Ookawa, T., Hobo, T., Yano, M., Murate, K., Ando, T., Miure, H., Asno, K., Ochiai, Y., Ikeda M., Nishitani, R., Ebistani, T., Ozaki, I., Angeles, E.R., Hirasana, T and Matsuoka, M 2010. New approach for rice improvement using a pleiotropic QTL gene for lodging resistance and yield. Nature Communications http://nature.com.

Setter, T.L., Laureles, E.V and Mazaredo, A.M. 1997. Lodging reduces yield of rice by self-shading and reductions in canopy photosynthesis. Field Crops Research. 49: 95-106.

SES, IRRI 2002. Standard Evaluation System for Rice. International Rice Research Institute, Philippines. 
Van Berloo, R. 1999.GGT: Software for the display of graphical genotypes. Journal of heredity. 90(2): 328-329.

Wu, L.L., Liu, Z., Jun-Min, W., Cong-Yi, Z and Kun-Ming, C. 2011. Morphological, anatomical, and physiological characteristics involved in development of the large culm trait in rice. Australian Journal of Crop Science. 5(11): 1356-1363.

Yamamoto, K., Yamamoto, T., Sugiyama, C., Hirasawa, T and Ookawa, T. 2013. Estimation of the locus for strong culm traits, using reciprocal chromosome segment substitution lines derived from the cross between rice varieties, koshihikari and takanari. Proceedings of $7^{\text {th }} \quad$ International Rice Genetics Symposium, 5 - 8 November 2013, Manila, Philippines. $435 \mathrm{Pp}$.

Yano, K., Ookawa, T., Taiichiro, A., Aya, K., Ochiai, Y., Hirasawa, T., Ebitani, T., Takarada, T., Yano, M., Yamamoto, T., Fukuoka, S., Wu, J., Ando, T., Ordonio, R.L., Hirano, $\mathrm{K}$ and Matsuoka, M. 2014. Isolation of a novel lodging resistance QTL gene involved in strigolactone signaling and its pyramiding with a QTL gene involved in another mechanism. Molecular Plant. 8(2): 303-315.

Yang, H., Fang, X., He, H and Xie, Z.2012. Relationship of characteristics of culm construction to lodging resistance and yield of Fujian-bred super-rice cultivars. Chinese Journal of Eco Agriculture. 20: 909-913.

Zhang, X., Li, H., Li, W., Xu, Z., Chen, W., Zhang, W and Wang, W. 2009. The lodging resistance of erect panicle japonica rice in Northern China. Scientia Agricultura Sinica. 42(7): 2305-2313.

Zheng, K., Subudhi, P.K., Domingo, J., Magantay, G and Huang, N.1995. Rapid DNA isolation for marker assisted selection in rice breeding. Rice Genetics News Letter. 12: 255-258.

Zhu, L.H., Zhong, D.B., Xu, J.L., Yu, S.B and Li, Z.K. 2008. Differential expression of lodging resistance related QTLs in rice (Oryza sativa L.). Plant Science. 175: 898-905.

\section{How to cite this article:}

Girija Rani, M. and Satyanarayana, P.V. 2018. Selection of Lodging Resistant Lines in Early Generations Using Linked Molecular Markers and Phenotypic Traits in Rice. Int.J.Curr.Microbiol.App.Sci. 7(01): 1638-1650. doi: https://doi.org/10.20546/ijcmas.2018.701.199 